\title{
Etiological Analysis of Reactive Gastropathy in an Urban Population
}

\author{
Sindhura Kollia ${ }^{a}$ Amit Mori $^{\mathrm{b}}$ Simcha Weissman ${ }^{\mathrm{c}}$ Tej I. Mehta ${ }^{\mathrm{d}}$ Khoi \\ Paul Dang-Ho ${ }^{b}$ Jamil Shah $^{b}$ Manpreet Singh ${ }^{b} \quad$ Madhavi Reddy ${ }^{b}$ \\ Anand Suryanarayan ${ }^{\mathrm{e}}$
}

aDepartment of Medicine, NYU Grossman School of Medicine, New York, NY, USA; ${ }^{b}$ Department of Gastroenterology, The Brooklyn Hospital Center, Brooklyn, NY, USA; ' Department of Medicine, Hackensack University-Palisades Medical Center, North Bergen, NJ, USA; ${ }^{\mathrm{d} D e p a r t m e n t}$ of Medicine, University of South Dakota Sanford School of Medicine, Sioux Falls, SD, USA; 'Department of Gastroenterology, NYU Brooklyn Hospital, Brooklyn, NY, USA

\section{Keywords}

Reactive gastropathy - Bile acid reflux · Nonsteroidal antiinflammatory drugs

\begin{abstract}
Background: Reactive gastropathy ( $R G$ ) is an adaptive response to assaults of the gastric mucosa. Demographic information regarding $\mathrm{RG}$ as well as the coincidence of $\mathrm{RG}$ and gastrointestinal cancer are poorly characterized entities. $\mathbf{O b}$ jective: Herein, we aim to investigate relationships of RG to both modifiable and nonmodifiable risk factors, as well as conduct a stratified analysis by race in an ethnically diverse, urban population. Methods: In this retrospective study, we queried an urban hospital inpatient pathology database searching for patients with surgical gastric biopsies positive for RG between March 25, 2015, and March 25, 2016. Of the 728 patients with a final diagnosis of RG, 292 were selected based on strict inclusion and exclusion criteria. We explored risk factors and conducted a stratified analysis for associations based on patient demographics. Results: In this urban
\end{abstract}

karger@karger.com www.karger.com/gat

Karger $\stackrel{\text { ' }}{5}$

GOPEN ACCESS
(C) 2021 The Author(s)

Published by S. Karger AG, Basel

This article is licensed under the Creative Commons AttributionNonCommercial-NoDerivatives 4.0 International License (CC BY NC-ND) (http://www.karger.com/Services/OpenAccessLicense). Usage and distribution for commercial purposes as well as any distribution of modified material requires written permission. minority population, nonsteroidal anti-inflammatory drugs (NSAIDs) were the most common medication associated with RG (Fig. 1), as well as the most common cause of RG, followed by chronic bile reflux. In addition, significant differences in demographics and gastropathic characteristics associated with RG, stratified by ethnicity, were found (Fig. 2). Notably, Hispanics, African Americans, and Caucasians had the highest rate of concomitant RG and diabetes, hypertension, and tobacco/alcohol use, respectively. Conclusion: Our study indicated that NSAID usage is the most common cause of RG, followed by bile reflux-mediated mucosal injury, in an ethnically diverse urban US-based population. Of note, few patients had intestinal metaplasia, suggesting it to be a slow or negligent sequela of RG.

(c) 2021 The Author(s)

Published by S. Karger AG, Basel

Presented as an abstract at the 2017 ACG conference in Orlando on Oct 13-18. 


\section{Introduction}

Reactive gastropathy (RG) is an adaptive response to assaults of the gastric mucosa. There is a constellation of endoscopic and histologic findings characterized by foveolar hyperplasia, smooth muscle fibers in the lamina propria, and vasodilatation and congestion of mucosal capillaries in the absence of significant inflammation [1]. Previous literature theorized these changes were secondary to bile reflux, however more recently, a broad-spectrum of chemical injuries have been attributed to this reactive change $[1,2]$.

The clinical phenomenon of RG was first described among postgastrectomy patients, but now it is more commonly encountered in patients on chronic nonsteroidal anti-inflammatory drugs (NSAIDs) [3, 4]. While gastric cancer secondary to $\mathrm{RG}$ is uncommon, stump carcinoma has been reported in postgastrectomy patients. Bile reflux has been implicated in these cases due to a bile-related propensity toward dysplastic changes and eventual development of gastric cancer [5]. Demographic information on RG is lacking, however, analogous gastrointestinal phenomenon, such as Barrett's esophagus, are known genetic risk factors [6, 7]. Moreover, the coincidence of RG and other gastrointestinal diseases, such as gastrointestinal cancer, are poorly characterized.

To this end, we investigated causative and associative relationships of RG with both modifiable and nonmodifiable risk factors, for gastrointestinal diseases and conducted stratified analysis by race in an ethnically diverse, urban population. We hope this will allow clinicians to strategically identify high-risk individuals to diagnose and treat RG prior to carcinomatous changes as well as identify patients that might benefit from RG-related medication reconciliation.

\section{Materials and Methods}

A retrospective study within a hospital serving an ethnically diverse, urban population consisting of $33.4,29$, and $5 \%$ of black, Hispanic, and Asian patients, respectively, was performed. We quarried the hospital inpatient pathology database searching for patients with surgical gastric biopsies positive for RG between the time periods of March 25, 2015, to March 25, 2016. Of the 728 patients with a final diagnosis of RG, only 292 of their reports provided sufficient details to draw significant conclusions regarding RG. Exclusion criteria were strict and consisted of patient records that did not contain information regarding; age, sex, race, smoking status, drinking status, comorbidities, or medications used. Inclusion criteria were strict and consisted of patients with the histologic diagnosis of RG that contained records with all the previ- ously mentioned patient information. We explored nonmodifiable factors such as sex, race, and age as well as modifiable habits such as smoking and drinking. We also examined associations with comorbidities, medications, and biopsy findings.

\section{Results}

Of 292 patients, $65 \%(n=191)$ were females. Mean age was $61.7( \pm 16.0)$ years. In terms of comorbidities, $30.1 \%$ $(n=88)$ had diabetes mellitus, and $61.3 \%(n=179)$ were diagnosed with hypertension (Table 1).

Helicobacter pylori was present in $2.4 \%$ of patients with antral RG. $40.4 \%$ of patients were on NSAIDs, and $33.6 \%$ of patients used proton pump inhibitors (PPI). 12.0\% ( $n$ $=35)$ of patients were active smokers, and $16.1 \%(n=47)$ of patients were actively drinking alcohol (Tables 1,2 ). NSAIDs were the most common medication associated with RG (Fig. 1). After excluding identified causes of RG, the remaining patients were categorized as those with "RG of unknown etiology." We hypothesize that these patients with unknown etiology had chronic bile reflux causing histological changes identified as RG.

Significant differences were noted in the distributions of sex, smoking history, alcohol use history, NSAID use, PPI therapy, diabetes mellitus, hypertension, appendectomies, pancreaticoduodenectomies, hernia repairs, duodenitis, tissue architectural changes, gastropathy location, and by ethnicity (Fig. 2). Significant correlations to reactive gastropathy $(p>0.05)$ included diverticulosis, prokinetic agent use, abscesses, Schatzki's ring, and strictures.

\section{Discussion}

The geographical prevalence of RG is uniformly distributed across the USA [8]. Around $65.7 \%$ of patients diagnosed with RG were female [8]. Similar findings were found in our study $(66 \%)$. The prevalence of RG increased with age with a peak of $23.6 \%$ in females older than 90 years and a peak of $17.9 \%$ in males $80-89$ years [8]. In literature, the mean age of a reactive gastropathy diagnosis is 59.4 years compared to the mean age of $55 \pm 18$ years in our study [8].

Our results demonstrated that the highest prevalence of RG was present in African Americans and Hispanics and then Caucasians. All three groups reported high rates of concomitant RG with hypertension, diabetes, and tobacco/alcohol use, respectively, as possible significant contributors. In our study, gastric and esophageal cancers secondary to RG were not reported. H. pylori was present 
Table 1. Demographic characteristics of study population stratified by ethnicity

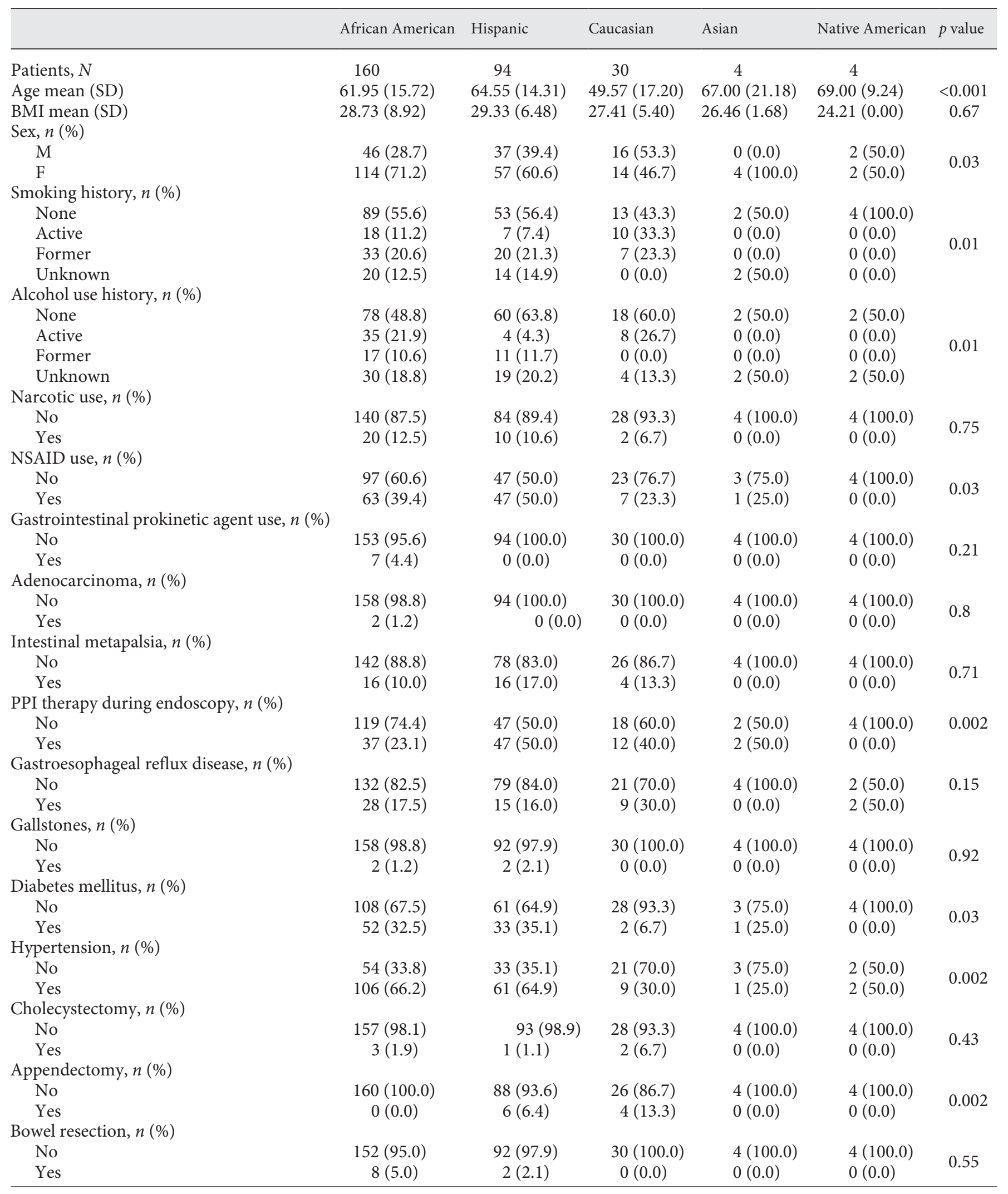


Table 1 (continued)

\begin{tabular}{|c|c|c|c|c|c|c|}
\hline & African American & Hispanic & Caucasian & Asian & Native American & $p$ value \\
\hline \multicolumn{7}{|c|}{ Gastrectomy, $n(\%)$} \\
\hline No & $157(98.1)$ & $92(97.9)$ & $28(93.3)$ & $4(100.0)$ & $4(100.0)$ & 0.6 \\
\hline \multicolumn{7}{|c|}{ Roux-en-Y gastrojejunostomy, $n(\%)$} \\
\hline No & $156(97.5)$ & $94(100.0)$ & $30(100.0)$ & $4(100.0)$ & $4(100.0)$ & \multirow{2}{*}{0.5} \\
\hline Yes & $4(2.5)$ & $0(0.0)$ & $0(0.0)$ & $0(0.0)$ & $0(0.0)$ & \\
\hline No & $160(100.0)$ & $94(100.0)$ & $28(93.3)$ & $4(100.0)$ & $4(100.0)$ & \multirow{2}{*}{0.001} \\
\hline Yes & $0(0.0)$ & $0(0.0)$ & $2(6.7)$ & $0(0.0)$ & $0(0.0)$ & \\
\hline \multicolumn{7}{|c|}{ Hernia repair, $n(\%)$} \\
\hline No & $160(100.0)$ & $92(97.9)$ & $30(100.0)$ & $3(75.0)$ & $4(100.0)$ & \multirow{2}{*}{$<0.001$} \\
\hline Yes & $0(0.0)$ & $2(2.1)$ & $0(0.0)$ & $1(25.0)$ & $0(0.0)$ & \\
\hline
\end{tabular}

NSAID, nonsteroidal anti-inflammatory drug.

Table 2. Gastropathic characteristics of the study population stratified by ethnicity

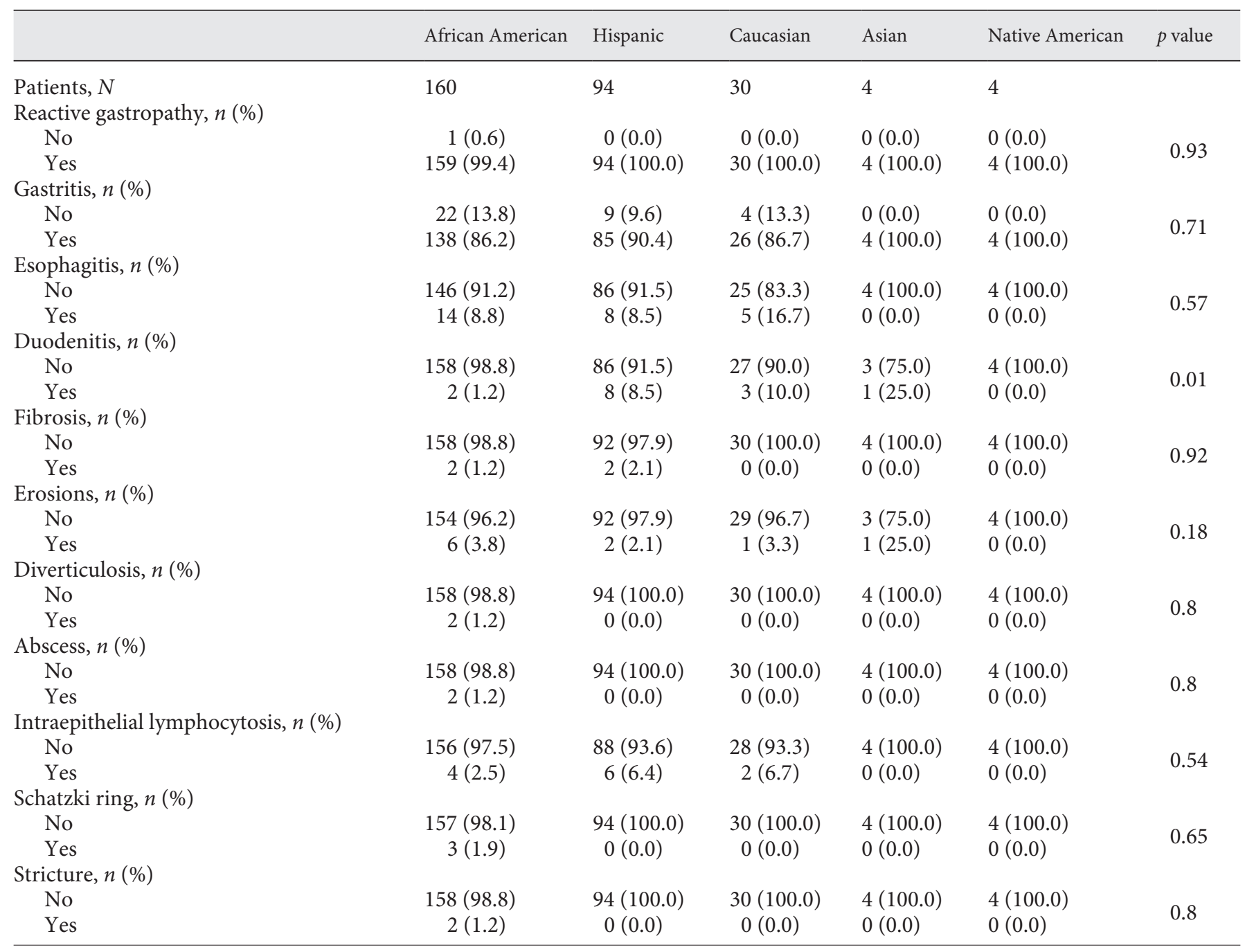


Table 2 (continued)

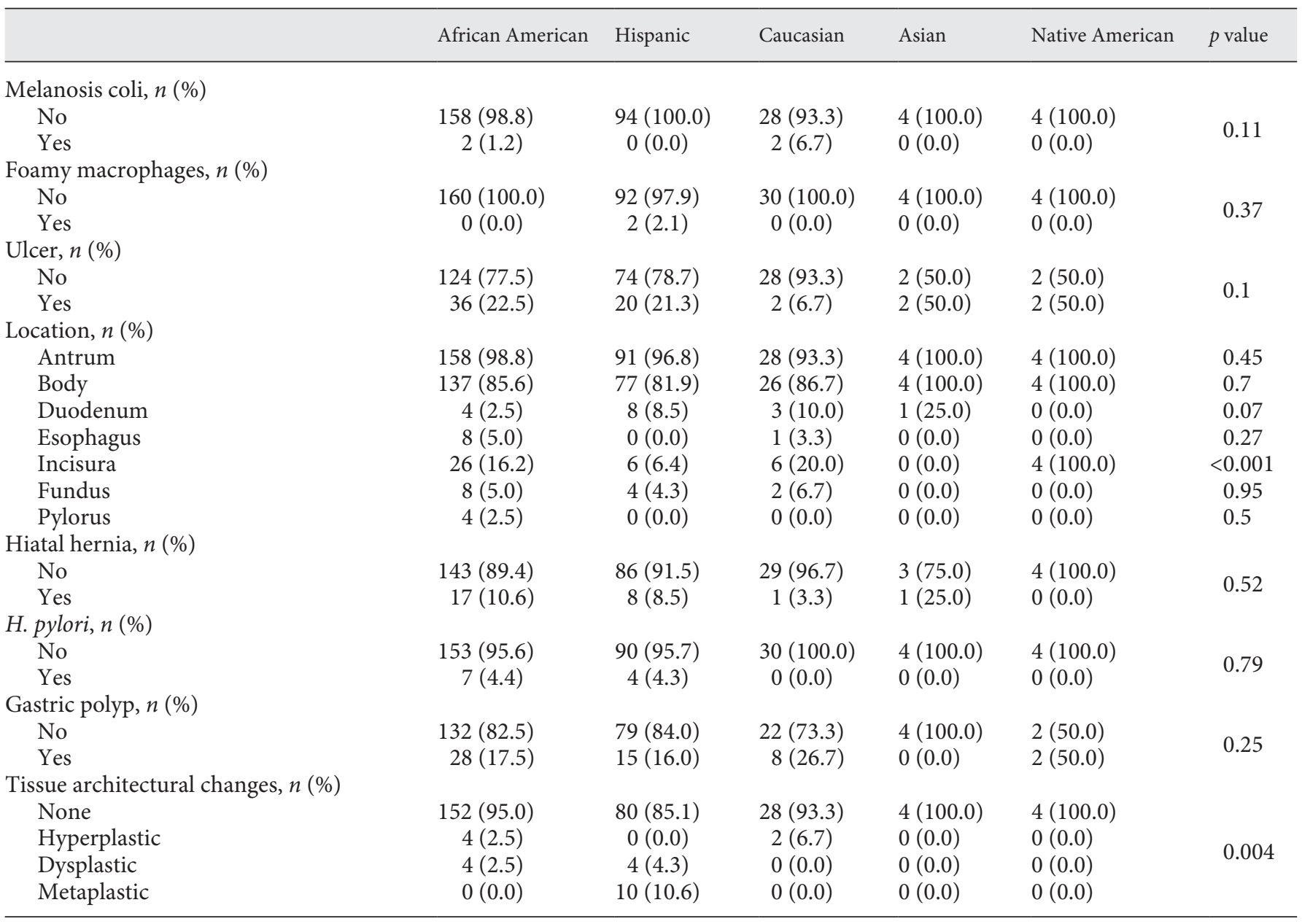

in Native Americans, Asians, and Caucasians. These findings differ from current literature which demonstrates a high prevalence of $H$. pylori in Hispanics and Native American/Alaskan Native. These groups along with Asian/Pacific Islanders had the highest incidence of upper GI symptoms from $H$. pylori-induced RG, while African Americans were at the highest risk for $H$. pyloriinduced gastrointestinal cancer [9]. These findings might differ due to our smaller groups of Caucasian, Asian, and Native American populations included in this study.

Various studies have demonstrated that patients with chronic NSAID use were more likely to develop RG $[1,8$, 10]. 30.3\% of patients in our study that developed RG were on NSAIDs. Proposed manifestations of NSAID-induced pathologies have shown to be associated with the patients that develop RG. Active pathologies such as peptic duode- nitis, duodenal intraepithelial lymphocytosis, active ileitis, focal active colitis, and collagenous colitis were included [8]. Bile reflux injury has been proposed as a mechanism leading to the development of RG as patients with reflux esophagitis and Barrett's esophagus, with or without dysplasia, was more likely to be diagnosed with RG [8].

In conclusion, NSAID usage had a high concurrent prevalence in patients with RG with bile reflux-mediated mucosal injury as a close second. While this shows correlation, further larger studies would need to be undertaken to analyze causation. Very few patients had intestinal metaplasia, suggesting it to be a slow or negligent sequelae of RG. Larger studies need to be done to evaluate the role of bile and other gastrointestinal pathologies involved in the causation of RG. 


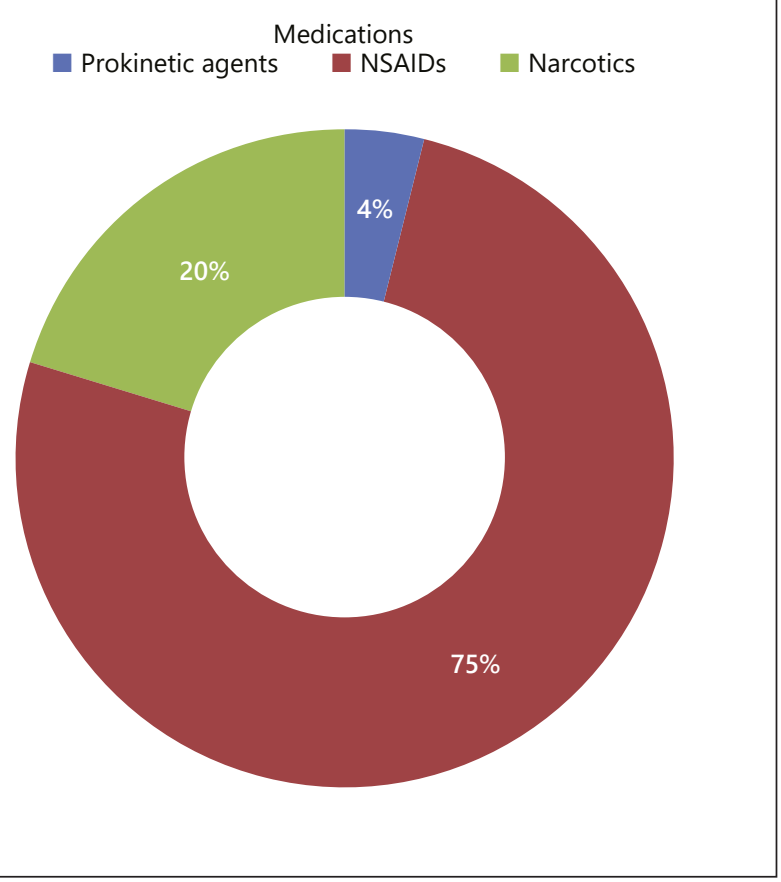

Fig. 1. Medications associated with RG in an urban minority patient population. RG, reactive gastropathy; NSAID, nonsteroidal anti-inflammatory drug.

\section{Statement of Ethics}

Written informed consent was obtained to publish this case as well as include any images for publication. Ethical considerations were taken into account according to the principles that comply with the guidelines for human studies. In addition, the research was conducted ethically in accordance with the World Medical Association Declaration of Helsinki. No funding, sponsors, institutional affiliations, potential conflicts of interest, incentives for subjects, and information exist within.

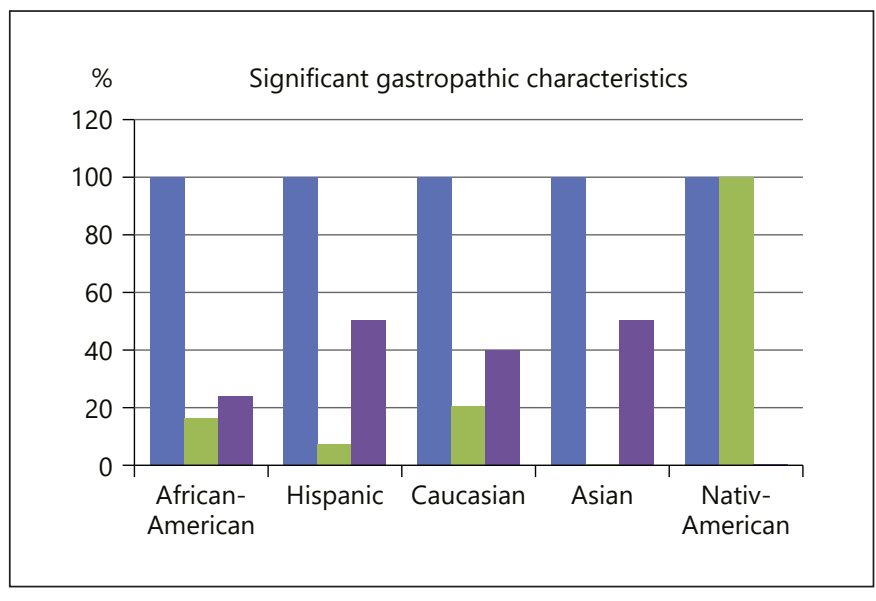

Fig. 2. Significant characteristics associated with RG stratified by ethnicity. RG, reactive gastropathy.

\section{Conflict of Interest Statement}

The authors have no conflicts of interest to declare.

\section{Funding Sources}

No funding was received for any part of the preparation or submission of this manuscript.

\section{Author Contributions}

S.K., S.W., A.M., T.I.M., K.P.D.H., and J.S. assisted with manuscript preparation and editing. M.R., A.S., and M.S. drafted and critically revised the manuscript. S.K. is the article guarantor. All the authors agree to the submission of this manuscript.

\section{References}

1 Sobala GM, King RF, Axon AT, Dixon MF. Reflux gastritis in the intact stomach. J Clin Pathol. 1990;43(4):303-6.

2 Quinn CM, Bjarnason I, Price AB. Gastritis in patients on non-steroidal anti-inflammatory drugs. Histopathology. 1993;23(4):341-8.

3 Burden WR, Hodges RP, Hsu M, O'Leary JP. Alkaline reflux gastritis. Surg Clin North Am. 1991;71(1):33-44.

4 Wolfe MM, Lichtenstein DR, Singh G. Gastrointestinal toxicity of nonsteroidal antiinflammatory drugs. N Engl J Med. 1999; 340(24):1888-99.
5 Johannesson KA, Hammar E, Staël von Holstein C. Mucosal changes in the gastric remnant: long-term effects of bile reflux diversion and Helicobacter pylori infection. Eur J Gastroenterol Hepatol. 2003;15(1):35-40.

6 Nwokediuko SC. Current trends in the management of gastroesophageal reflux disease: a review. ISRN Gastroenterol. 2012;2012: 391631.

7 Nordenstedt H, Lagergren J. Environmental factors in the etiology of gastroesophageal reflux disease. Expert Rev Gastroenterol Hepatol. 2008;2(1):93-103.
8 Maguilnik I, Neumann WL, Sonnenberg A, Genta RM. Reactive gastropathy is associated with inflammatory conditions throughout the gastrointestinal tract. Aliment Pharmacol Ther. 2012;36(8):736-43.

9 Huerta-Franco MR, Banderas JW, Allsworth JE. Ethnic/racial differences in gastrointestinal symptoms and diagnosis associated with the risk of Helicobacter pylori infection in the US. Clin Exp Gastroenterol. 2018;11:39-49.

10 Taha AS, Nakshabendi I, Lee FD, Sturrock $\mathrm{RD}$, Russell RI. Chemical gastritis and Helicobacter pylori related gastritis in patients receiving non-steroidal anti-inflammatory drugs: comparison and correlation with peptic ulceration. J Clin Pathol. 1992;45(2):135-9. 